\title{
ON THE VOLUME BOUND IN THE DVORETZKY-ROGERS LEMMA
}

\author{
FERENC FODOR, MÁRTON NASZÓDI, AND TAMÁS ZARNÓCZ
}

\begin{abstract}
The classical Dvoretzky-Rogers lemma provides a deterministic algorithm by which, from any set of isotropic vectors in Euclidean $d$-space, one can select a subset of $d$ vectors whose determinant is not too small. Subsequently, Pelczyński and Szarek improved this lower bound by a factor depending on the dimension and the number of vectors.

Pivovarov, on the other hand, determined the expectation of the square of the volume of parallelotopes spanned by $d$ independent random vectors in $\mathbb{R}^{d}$, each one chosen according to an isotropic measure. We extend Pivovarov's result to a class of more general probability measures, which yields that the volume bound in the Dvoretzky-Rogers lemma is, in fact, equal to the expectation of the squared volume of random parallelotopes spanned by isotropic vectors. This allows us to give a probabilistic proof of the improvement of Pelczyński and Szarek, and provide a lower bound for the probability that the volume of such a random parallelotope is large.
\end{abstract}

\section{INTRODUCTION}

Given a set of isotropic vectors in Euclidean $d$-space $\mathbb{R}^{d}$ (see definition below), the Dvoretzky-Rogers lemma states that one may select a subset of $d$ "well spread out" vectors. As a consequence, the determinant of these $d$ vectors is at least $\sqrt{d ! / d^{d}}$. This selection is deterministic: we start with an arbitrary element of the set, and then select more vectors one-by-one in a certain greedy manner.

Pivovarov Piv10, Lemma 3, p. 49], on the other hand, chooses $d$ vectors randomly and then computes the expectation of the square of the resulting determinant. In this note, we extend Pivovarov's result to a wider class of measures, and apply this extension to obtain the improved lower bound of Pelczyński and Szarek, cf. PS91 Proposition 2.1, on the maximum of the volume of parallelotopes spanned by $d$ vectors from the support of the measure. Thus, we give a probabilistic interpretation of the volume bound in the Dvoretzky-Rogers lemma.

We denote the Euclidean scalar product by $\langle\cdot, \cdot\rangle$, the induced norm by $|\cdot|$. We use the usual notation $B^{d}$ for the unit ball of $\mathbb{R}^{d}$ centered at the origin $o$, and $S^{d-1}$ for its boundary bd $B^{d}$. We call a compact convex set $K \subset \mathbb{R}^{d}$ with non-empty interior a convex body. For detailed information on the properties of convex bodies, we refer to the books by Gruber Gru07] and Schneider [Sch14].

Let $\operatorname{Id}_{d}$ be the identity map on $\mathbb{R}^{d}$. For $u, v \in \mathbb{R}^{d}$, let $u \otimes v: \mathbb{R}^{d} \rightarrow \mathbb{R}^{d}$ denote the tensor product of $u$ and $v$, that is, $(u \otimes v)(x)=\langle v, x\rangle u$ for any $x \in \mathbb{R}^{d}$. Note that when $u \in S^{d-1}$ is a unit vector, $u \otimes u$ is the orthogonal projection to the linear subspace spanned by $u$.

For two functions $f(n), g(n)$, we use the notation $f(n) \sim g(n)($ as $n \rightarrow \infty)$ if $\lim _{n \rightarrow \infty} f(n) / g(n)=1$.

An isotropic measure is a probability measure $\mu$ on $\mathbb{R}^{d}$ with the following two properties.

$$
\int_{\mathbb{R}^{d}} x \otimes x \mathrm{~d} \mu(x)=\operatorname{Id}_{d}
$$

and the center of mass of $\mu$ is at the origin, that is,

$$
\int_{\mathbb{R}^{d}} x \mathrm{~d} \mu(x)=0 .
$$

Pivovarov Piv10 proved the following statement about the volume of random parallelotopes spanned by $d$ independent, isotropic vectors.

2010 Mathematics Subject Classification. 52A22,52B11,52A38,52A40.

Key words and phrases. isotropic vectors, John's theorem, Dvoretzky-Rogers lemma, volume, decomposition of the identity.

The final version of the paper was published in Pacific Journal of Mahtmematics 301 (2019), No. 1, 89-99. https://dx.doi.org/10.2140/pjm.2019.301.89. 
Lemma 1 (Pivovarov Piv10, Lemma 3). Let $x_{1}, \ldots, x_{d}$ be independent random vectors distributed according to the isotropic measures $\mu_{1}, \ldots, \mu_{d}$ in $\mathbb{R}^{d}$. Assume that $x_{1}, \ldots, x_{d}$ are linearly independent with probability 1. Then

$$
\mathbb{E}\left(\left[\operatorname{det}\left(x_{1}, \ldots, x_{d}\right)\right]^{2}\right)=d ! .
$$

We note that Lutwak, Yang and Zhang in LYZ04, §2] established similar results for the case of discrete isotropic measures, which could also be used to prove the volumetric bounds in Theorem 2 , see, for example, LYZ04, formula (2.5) on page 167].

We extend Lemma 1 to a more general class of measures in the following way.

Lemma 2. Let $x_{1}, \ldots, x_{d}$ be independent random vectors distributed according to the measures $\mu_{1}, \ldots, \mu_{d}$ in $\mathbb{R}^{d}$ satisfying (1). Assume that $\mu_{i}(\{0\})=0$ for $i=1, \ldots$, d. Then (3) holds.

We provide a simple and direct proof of Lemma 2 in Section 2.

Lemmas 1 and 2 yield the value of the second moment of the volume of random parallelotopes with isotropic generating vectors. On the other hand, Milman and Pajor [MP, §3.7] gave a lower bound for the $p$-th moment (with $0<p<2$ ) of this volume in the case when the generating vectors are selected according to the uniform distribution from an isotropic and origin-symmetric convex body; for more general results, cf. BGVV14, §3.5.1]. All of the previously mentioned results hold in expectation.

As a different approach, we mention Pivovarov's work Piv10, where lower bounds on the volume of a random parallelotope are shown to hold with high probability under the assumption that the measures are log-concave.

For more information on properties of random parallelotopes, and random polytopes in general, we refer to the book by Schneider and Weil [SW08], the survey by Schneider [Sch], and the references therein.

In this paper, our primary, geometric motivation in studying isotropic measures is the following celebrated theorem of John [Joh48, which we state in the refined form obtained by Ball Bal92] (see also [Bal97]).

Theorem 1. Let $K$ be a convex body in $\mathbb{R}^{d}$. Then there exists a unique ellipsoid of maximal volume contained in $K$. Moreover, this maximal volume ellipsoid is the $d$-dimensional unit ball $B^{d}$ if and only if there exist vectors $u_{1}, \ldots, u_{m} \in \mathrm{bd} K \cap S^{d-1}$ and (positive) real numbers $c_{1}, \ldots, c_{m}>0$ such that

$$
\sum_{i=1}^{m} c_{i} u_{i} \otimes u_{i}=\operatorname{Id}_{d},
$$

and

$$
\sum_{i=1}^{m} c_{i} u_{i}=0
$$

Note that taking the trace in (4) yields $\sum_{i=1}^{m} c_{i}=d$. Thus, the Borel measure $\mu_{K}$ on $\sqrt{d} S^{d-1}$ with $\operatorname{supp} \mu_{K}=\left\{\sqrt{d} u_{1}, \ldots, \sqrt{d} u_{m}\right\}$ and $\mu_{K}\left(\left\{\sqrt{d} u_{i}\right\}\right)=c_{i} / d(i=1, \ldots, m)$ is a discrete isotropic measure.

If a finite system of unit vectors $u_{1}, \ldots, u_{m}$ in $\mathbb{R}^{d}$, together with a set of positive weights $c_{1}, \ldots, c_{m}$ satisfies (4) and (5), then we say that it forms a John decomposition of the identity. For each convex body $K$, there exists an affine image $K^{\prime}$ of $K$ for which the maximal volume ellipsoid contained in $K^{\prime}$ is $B^{d}$, and $K^{\prime}$ is unique up to orthogonal transformations of $\mathbb{R}^{d}$.

The classical lemma of Dvoretzky and Rogers DR50 states that in a John decomposition of the identity, one can always find $d$ vectors such that the selected vectors are not too far from an orthonormal system.

Lemma 3 (Dvoretzky-Rogers lemma DR50). Let $u_{1}, \ldots, u_{m} \in S^{d-1}$ and $c_{1}, \ldots, c_{m}>0$ such that (4) holds. Then there exists an orthonormal basis $b_{1}, \ldots, b_{d}$ of $\mathbb{R}^{d}$ and a subset $\left\{x_{1}, \ldots, x_{d}\right\} \subset\left\{u_{1}, \ldots, u_{m}\right\}$ with $x_{j} \in \operatorname{lin}\left\{b_{1}, \ldots, b_{j}\right\}$ and

$$
\sqrt{\frac{d-j-1}{d}} \leq\left\langle x_{j}, b_{j}\right\rangle \leq 1
$$

for $j=1, \ldots, d$. 
Consider the parallelotope $P$ spanned by the selected $d$ vectors $x_{1}, \ldots, x_{d}$. The volume of $P$ is bounded from below by

$$
(\operatorname{Vol}(P))^{2}=\left[\operatorname{det}\left(x_{1}, \ldots, x_{d}\right)\right]^{2} \geq \frac{d !}{d^{d}} .
$$

Our study of (7) is motivated in part by the recent proof [Nas16] of a conjecture of Bárány, Katchalski and Pach, where this bound is heavily relied on.

The main results of this paper are the following two statements. Theorem 2 is essentialy the same as Proposition 2.1 of Pelczyński and Szarek PS91, however, here we give a probabilistic proof and interpretation. In Theorem 2 (iii) and (iii), we also note that when $m$ is small the improvement on the original Dvoretzky-Rogers bound is larger.

Theorem 2. Let $u_{1}, \ldots, u_{m} \in S^{d-1}$ be unit vectors satisfying (4) with some $c_{1}, \ldots, c_{m}>0$. Then there is a subset $\left\{x_{1}, \ldots, x_{d}\right\} \subset\left\{u_{1}, \ldots, u_{m}\right\}$ with

$$
\left[\operatorname{det}\left(x_{1}, \ldots, x_{d}\right)\right]^{2} \geq \gamma(d, \bar{m}) \cdot \frac{d !}{d^{d}},
$$

where $\gamma(d, \bar{m})=\frac{\bar{m}^{d}}{d !}\left(\begin{array}{c}\bar{m} \\ d\end{array}\right){ }^{-1}$, and $\bar{m}=\min \{m, d(d+1) / 2\}$.

Moreover, for $\gamma(d, \bar{m})$, we have

(i) $\gamma(d, \bar{m}) \geq \gamma(d, d(d+1) / 2) \geq 3 / 2$ for any $d \geq 2$ and $m \geq d$. And $\gamma(d, d(d+1) / 2)$ is monotonically increasing, and $\lim _{d \rightarrow \infty} \gamma(d, d(d+1) / 2)=e$.

(ii) Fix a $c>1$, and consider the case when $m \leq c d$ with $c \geq 1+1 / d$. Then

$$
\gamma(d, m) \geq \gamma(d,\lceil c d\rceil) \sim \sqrt{\frac{c-1}{c}}\left(\frac{c-1}{c}\right)^{(c-1) d} e^{d}, \quad \text { as } d \rightarrow \infty .
$$

(iii) Fix an integer $k \geq 1$, and consider the case when $m \leq d+k$. Then

$$
\gamma(d, m) \geq \gamma(d, d+k) \sim \frac{k ! e^{k}}{\sqrt{2 \pi}} \frac{e^{d}}{(d+k)^{k+1 / 2}}, \text { as } d \rightarrow \infty .
$$

We note that in (ii) and (iii), the improvements are exponentially large in $d$ as $d$ tends to infinity.

The following statement provides a lower bound on the probability that $d$ independent, identically distributed random vectors selected from $\left\{u_{1}, \ldots, u_{m}\right\}$ according to the distribution determined by the weights $\left\{c_{1}, \ldots, c_{m}\right\}$ has large volume.

Proposition 1. Let $\lambda \in(0,1)$. With the notations and assumptions of Theorem 2, if we choose the vectors $x_{1}, \ldots, x_{d}$ independently according to the distribution $\mathbb{P}\left(x_{\ell}=u_{i}\right)=c_{i} / d$ for each $\ell=1, \ldots, d$ and $i=$ $1, \ldots, m$, then with probability at least $(1-\lambda) e^{-d}$, we have that

$$
\left[\operatorname{det}\left(x_{1}, \ldots, x_{d}\right)\right]^{2} \geq \lambda \gamma(d, \bar{m}) \cdot \frac{d !}{d^{d}} .
$$

The geometric interpretation of Theorem 2 is the following. If $K$ is a convex polytope with $n$ facets, and $B^{d}$ is the maximal volume ellipsoid in $K$, then the number of contact points $u_{1}, \ldots, u_{m}$ in John's theorem is at most $m \leq n$. Thus, Theorem 2 yields a simplex in $K$ of not too small volume, with one vertex at the origin.

In particular, consider $k=1$ in Theorem 2 (iii), that is, when $K$ is the regular simplex whose inscribed ball is $B^{d}$. Then the John decomposition of the identity determined by $K$ consists of $d+1$ unit vectors that determine the vertices of a regular $d$-simplex inscribed in $B^{d}$, which we denote by $\Delta_{d}$, and note that $\operatorname{Vol}\left(\Delta_{d}\right)=(d+1)^{\frac{d+1}{2}} /\left(d^{d / 2} d\right.$ !). Clearly, in this John decomposition of the identity, the volume of the simplex determined by any $d$ of the vectors $u_{1}, \ldots, u_{d+1}$ is

$$
\operatorname{Vol}\left(\Delta_{d}\right) /(d+1)=\frac{(d+1)^{\frac{d-1}{2}}}{d^{d / 2} d !} .
$$

By Theorem 2, we obtain that

$$
\max \left[\operatorname{det}\left(u_{i_{1}}, \ldots, u_{i_{d}}\right)\right]^{2} \geq \frac{(d+1)^{d-1}}{d !} \cdot \frac{d !}{d^{d}}=\frac{(d+1)^{d-1}}{d^{d}},
$$


which yields the same bound for the largest volume simplex as the right-hand-side of (8). Thus, Theorem 2 is sharp in this case.

We will use the following theorem in our argument.

Theorem 3 ([Joh48, Peł90, Bal92, GS05]). If a set of unit vectors satisfies (4) (resp., (4) and (5)) with some positive scalars $c_{i}^{\prime}$, then a subset of $m$ elements also satisfies (4) (resp., (4) and (5) with some positive scalars $c_{i}$, where

$$
d+1 \leq m \leq d(d+1) / 2
$$

(resp., $d+1 \leq m \leq d(d+3) / 2)$.

In Section 4, we outline a proof of Theorem 3 for two reasons. First, we will use the part when only (4) is assumed, which is only implicitly present in [GS05]. Second, in GS05], the result is described in terms of the contact points of a convex body with its maximal volume ellipsoid, that is, in the context of John's theorem. We, on the other hand, would like to give a presentation where the linear algebraic fact and its use in convex geometry are separated. Nevertheless, our proof is very close to the one given in [GS05].

\section{Proof of Lemma 2}

The idea of the proof is to slightly rotate each distribution so that the probability that the $d$ vectors are linearly independent is 1 . Then we may apply Pivovarov's lemma, and use a limit argument as the $d$ rotations each tend to the identity.

Let $A_{1}, \ldots, A_{d}$ be matrices in $S O(d)$ chosen independently of each other and of the $x_{i}$ s according to the unique Haar probability measure on $S O(d)$. Fix an arbitrary non-zero unit vector $e$ in $\mathbb{R}^{d}$. Note that $A_{i} x_{i} /\left|x_{i}\right|$ and $A_{i} e$ have the same distribution: both are uniformly chosen points of the unit sphere according to the uniform probability distribution on $S^{d-1}$. A bit more is true: the joint distribution of $A_{1} x_{1} /\left|x_{1}\right|, \ldots, A_{d} x_{d} /\left|x_{d}\right|$ and the joint distribution of $A_{1} e, \ldots, A_{d} e$ are the same: they are independently chosen, uniformly distributed points on the unit sphere. It follows that

$$
\mathbb{P}\left(A_{1} x_{1}, \ldots, A_{d} x_{d} \text { are lin. indep. }\right)=\mathbb{P}\left(A_{1} e, \ldots, A_{d} e \text { are lin. indep. }\right)=1 .
$$

Denote the Haar measure on $Z:=S O(d)^{d}$ by $\nu$. Thus, we have

$$
\begin{gathered}
1=\mathbb{P}\left(A_{1} x_{1}, \ldots, A_{d} x_{d} \text { are lin. indep. }\right)= \\
\int_{Z} \int_{\mathbb{R}^{d}} \int_{\mathbb{R}^{d}} \ldots \int_{\mathbb{R}^{d}} \mathbb{1}_{\left\{A_{1} x_{1}, \ldots, A_{d} x_{d} \text { are lin. indep. }\right\}}\left(x_{1}, \ldots, x_{d}, A_{1}, \ldots, A_{d}\right) \\
\mathrm{d} \mu_{1}\left(x_{1}\right) \ldots \mathrm{d} \mu_{d}\left(x_{d}\right) \mathrm{d} \nu\left(A_{1}, \ldots, A_{d}\right) \\
=\int_{Z} \mathbb{P}\left(A_{1} x_{1}, \ldots, A_{d} x_{d} \text { are lin. indep. } \mid A_{1}, \ldots, A_{d}\right) \mathrm{d} \nu\left(A_{1}, \ldots, A_{d}\right),
\end{gathered}
$$

where $\mathbb{1}$ denotes the indicator function.

Thus,

$$
1=\mathbb{P}\left[\mathbb{P}\left(A_{1} x_{1}, \ldots, A_{d} x_{d} \text { are lin. indep. } \mid A_{1}, \ldots, A_{d}\right)=1\right] .
$$

We call a $d$-tuple $\left(A_{1}, \ldots, A_{d}\right) \in Z$ 'good' if $A_{1} x_{1}, \ldots, A_{d} x_{d}$ are linearly independent with probability 1 . In (10), we obtained that the set of not good elements of $Z$ is of measure zero.

Thus, we may choose a sequence $\left(A_{1}^{(j)}, A_{2}^{(j)}, \ldots, A_{d}^{(j)}\right), j=1,2, \ldots$ in $Z$, such that $\left\|A_{i}^{(j)}-\operatorname{Id}_{d}\right\|<1 / j$ for all $i$ and $j$, and $\left(A_{1}^{(j)}, \ldots, A_{d}^{(j)}\right)$ is good for each $j$.

Note that for any $j$,

$$
\left[\operatorname{det}\left(A_{1}^{(j)} x_{1}, \ldots, A_{d}^{(j)} x_{d}\right)\right]^{2} \leq\left|A_{1}^{(j)} x_{1}\right|^{2}\left|A_{2}^{(j)} x_{2}\right|^{2} \ldots\left|A_{d}^{(j)} x_{d}\right|^{2},
$$

and

$$
\mathbb{E}\left[\left|A_{1}^{(j)} x_{1}\right|^{2}\left|A_{2}^{(j)} x_{2}\right|^{2} \ldots\left|A_{d}^{(j)} x_{d}\right|^{2}\right]=d^{d}
$$

We conclude that 


$$
\begin{gathered}
\mathbb{E}\left(\left[\operatorname{det}\left(x_{1}, \ldots, x_{d}\right)\right]^{2}\right)= \\
\mathbb{E}\left(\left[\operatorname{det} \lim _{j \rightarrow \infty}\left(A_{1}^{(j)} x_{1}, \ldots, A_{d}^{(j)} x_{d}\right)\right]^{2}\right) \stackrel{(a)}{=} \\
\mathbb{E}\left(\left[\lim _{j \rightarrow \infty} \operatorname{det}\left(A_{1}^{(j)} x_{1}, \ldots, A_{d}^{(j)} x_{d}\right)\right]^{2}\right) \stackrel{(b)}{=} \\
\lim _{j \rightarrow \infty} \mathbb{E}\left(\left[\operatorname{det}\left(A_{1}^{(j)} x_{1}, \ldots, A_{d}^{(j)} x_{d}\right)\right]^{2}\right),
\end{gathered}
$$

where, in (a), we use that the determinant is continuous. In (b), Lebesgue's Dominated Convergence Theorem may be applied by (11) and 12 .

Fix $j$ and let $y_{1}=A_{1}^{(j)} x_{1}, \ldots, y_{d}=A_{d}^{(j)} x_{d}$. In order to emphasize that the assumption (2) is not needed, and also for completeness, we repeat Pivovarov's argument. For $k=1, \ldots, d-1$, let $P_{k}$ denote the orthogonal projection of $\mathbb{R}^{d}$ onto the linear subspace $\operatorname{span}\left\{y_{1}, \ldots, y_{k}\right\}^{\perp}$. Thus,

$$
\left|\operatorname{det}\left(y_{1}, \ldots, y_{d}\right)\right|=\left|y_{1}\right|\left|P_{1} y_{2}\right| \cdots\left|P_{d-1} y_{d}\right| \text {. }
$$

Note that with probability $1, \operatorname{rank} P_{k}=d-k$. It follows from (1) that $\mathbb{E}\left|P_{k} y_{k+1}\right|^{2}=d-k$. Fubini's Theorem applied to 13 completes the proof of Lemma 2.

\section{Proofs of Theorem 2 and Proposition 1}

Let $u_{1}, \ldots u_{m} \in S^{d-1}$ be a set of vectors satisfying (4) with some positive weights $c_{1}, \ldots, c_{m}$. We set the probability of each vector $u_{i}, i=1, \ldots m$ as $p_{i}=c_{i} / d$, and obtain a discrete probability distribution.

Let $u_{i_{1}}, \ldots, u_{i_{d}}$ be independent random vectors from the set $u_{1}, \ldots, u_{m}$ chosen (with possible repetitions) according to the above probability distribution.

By Lemma 2, we have that

$$
\mathbb{E}\left(\left[\operatorname{det}\left(u_{i_{1}}, \ldots, u_{i_{d}}\right)\right]^{2}\right)=\frac{d !}{d^{d}} .
$$

Since the probability that the random vectors $u_{i_{1}}, \ldots, u_{i_{d}}$ are linearly dependent is positive,

$$
\max \left[\operatorname{det}\left(u_{i_{1}}, \ldots, u_{i_{d}}\right)\right]^{2}>\frac{d !}{d^{d}} .
$$

Our goal is to quantify this inequality by bounding from below the probability that the determinant is 0 . Let

$$
M^{2}:=\max \left[\operatorname{det}\left(u_{i_{1}}, \ldots, u_{i_{d}}\right)\right]^{2} .
$$

Note that if an element of $\left\{u_{1}, \ldots, u_{m}\right\}$ is selected at least twice, then $\operatorname{det}\left(u_{i_{1}}, \ldots, u_{i_{d}}\right)=0$. Thus,

$$
\mathbb{E}\left(\left[\operatorname{det}\left(u_{i_{1}}, \ldots, u_{i_{d}}\right)\right]^{2}\right) \leq M^{2} P_{1},
$$

where $P_{1}$ denotes the probability that all indices are pairwise distinct. Therefore,

$$
M^{2} \geq \frac{d !}{d^{d}} \cdot \frac{1}{P_{1}} .
$$

Note that $P_{1}$ is a degree $d$ elementary symmetric function of the variables $p_{1}, \ldots, p_{m}$. Furthermore, $p_{1}+\ldots+p_{m}=1$ and $p_{i} \geq 0$ for all $i=1, \ldots, m$. It can easily be seen (using Lagrange multipliers, or by induction on $m$ ) that for fixed $m$ and $d$, the maximum of $P_{1}$ is attained when $p_{1}=\ldots=p_{m}=1 / m$. Thus,

$$
P_{1} \leq d !\left(\begin{array}{c}
m \\
d
\end{array}\right) \frac{1}{m^{d}} .
$$

In summary,

$$
M^{2} \geq \frac{d !}{d^{d}} \cdot \frac{m^{d}}{d !}\left(\begin{array}{c}
m \\
d
\end{array}\right)^{-1}
$$

First, we note that $\gamma(d, m):=\frac{m^{d}}{d !}\left(\begin{array}{c}m \\ d\end{array}\right)^{-1}$ is decreasing in $m$. Thus, by (9), we may assume that $m$ is as large as possible, that is, $m=\frac{d(d+1)}{2}$ proving the first part of Theorem 2 . 
3.1. Proof of Theorem 2 (i). Let $\gamma(d):=\gamma(d, d(d+1) / 2)$. We show that $\gamma(d)$ is increasing in $d$.

With the notation $m:=d(d+1) / 2$, we note that $(d+1)(d+2) / 2=m+d+1$. Thus,

$$
\frac{\gamma(d+1)}{\gamma(d)}=\frac{(m+d+1)^{d+1} m \cdots(m-d+1)}{m^{d}(m+d+1) \cdots(m+1)}=\frac{(m+d+1)^{d}}{m^{d}} \cdot \frac{m \cdots(m-d+1)}{(m+d) \cdots(m+1)}
$$

Thus, we need to show that

$$
1+\frac{d+1}{m}>\sqrt[d]{\left(1+\frac{d}{m}\right)\left(1+\frac{d}{m-1}\right) \cdots\left(1+\frac{d}{m-d+1}\right)},
$$

which, by the AM/GM inequality follows, if

which is equivalent to

$$
1+\frac{d+1}{m} \geq 1+d \frac{\frac{1}{m}+\frac{1}{m-1}+\ldots+\frac{1}{m-d+1}}{d},
$$

$$
\frac{d}{m} \geq \frac{1}{m-1}+\frac{1}{m-2}+\ldots+\frac{1}{m-d+1} .
$$

For this to hold, it is sufficient to show that for every integer or half of an integer $1 \leq i \leq d / 2$, we have that

$$
\frac{2 d}{(d-1) m} \geq \frac{1}{m-i}+\frac{1}{m-d+i} .
$$

After substituting $m=d(d+1) / 2$, it is easy to see that 14 holds.

Finally, $\lim _{d \rightarrow \infty} \gamma(d)=e$ follows from Stirling's formula.

3.2. Proof of Theorem 2 (ii) and (iii). Stirling's formula yields both claims.

3.3. Proof of Proposition 1. Let $X$ denote the random variable $X:=\left[\operatorname{det}\left(x_{1}, \ldots, x_{d}\right)\right]^{2}, E:=\mathbb{E}(X)=\frac{d !}{d^{d}}$, and $q:=\mathbb{P}\left(X \geq \frac{\lambda E}{P_{1}}\right)$, where, as in the proof of Theorem 2, $P_{1}:=\mathbb{P}\left(x_{1}, \ldots, x_{d}\right.$ are pairwise distinct $)$.

In the proof of Theorem 2, we established

$$
P_{1} \leq(\gamma(d, \bar{m}))^{-1}, \text { and thus, } q \leq \mathbb{P}\left(\left[\operatorname{det}\left(x_{1}, \ldots, x_{d}\right)\right]^{2} \geq \lambda \gamma(d, \bar{m}) \cdot \frac{d !}{d^{d}}\right) .
$$

Using the fact that $X$ is at most one, we have

$$
E \leq \frac{\lambda E}{P_{1}} \mathbb{P}\left(X<\frac{\lambda E}{P_{1}} \text { and } x_{1}, \ldots, x_{d} \text { are pairwise distinct }\right)+\mathbb{P}\left(X \geq \frac{\lambda E}{P_{1}}\right) .
$$

That is, $E \leq \frac{\lambda E}{P_{1}}\left(P_{1}-q\right)+q$, and thus, by 15

$$
q \geq \frac{(1-\lambda) E}{1-\frac{\lambda E}{P_{1}}} \geq \frac{(1-\lambda) d !}{d^{d}-\lambda \gamma(d, \bar{m}) d !} \geq(1-\lambda) e^{-d}
$$

completing the proof of Proposition 1

\section{Proof of Theorem 3}

First, observe that (4) holds with some positive scalars $c_{i}$, if and only if, the matrix $\operatorname{Id}_{d} / d$ is in the convex hull of the set $\mathcal{A}=\left\{v_{i} \otimes v_{i}: i=1, \ldots, m\right\}$ in the real vector space of $d \times d$ matrices. The set $\mathcal{A}$ is contained in the subspace of symmetric matrices with trace 1 , which is of dimension $d(d+1) / 2-1$. Carathéodory's theorem [Sch14, Theorem 1.1.4] now yields the desired upper bound on $m$.

In the case when both (4) and (5) are assumed, we lift our vectors into $\mathbb{R}^{d+1}$ as follows. Let $\hat{v}_{i}=$ $\sqrt{\frac{d}{d+1}}\left(v_{i}, 1 / \sqrt{d}\right) \in \mathbb{R}^{d+1}$. It is easy to check that $\left|\hat{v}_{i}\right|=1$, and that (4) holds for the vectors $\hat{v}_{i}$ with some positive scalars $\hat{c}_{i}$ if, and only if, (4) and (5) hold for the vectors $v_{i}$ with scalars $c_{i}=\frac{d}{d+1} \hat{c}_{i}$. Now, $\hat{v}_{i} \otimes \hat{v}_{i}$, $i=1, \ldots, m$ are symmetric $(d+1) \times(d+1)$ matrices of trace one, and their $(d+1, d+1)$ th entry is $1 /(d+1)$. 
The dimension of this subspace of $\mathbb{R}^{(d+1) \times(d+1)}$ is $d(d+3) / 2-1$, thus, again, by Carathéodory's theorem, the proof is complete.

\section{AcKnowledgements}

F. Fodor and T. Zarnócz are supported in part by Hungarian National Research, Development and Innovation Office NKFIH grant K 116451.

M. Naszódi was partially supported by the National Research, Development and Innovation Office (NKFIH) grant NKFI-K119670 and by the ÚNKP-17-4 New National Excellence Program of the Ministry of Human Capacities.

\section{REFERENCES}

[Bal92] K. Ball, Ellipsoids of maximal volume in convex bodies, Geom. Dedicata 41 (1992), no. 2, 241-250.

[Bal97] K. Ball, An elementary introduction to modern convex geometry, Flavors of geometry, Math. Sci. Res. Inst. Publ., vol. 31, Cambridge Univ. Press, Cambridge, 1997, pp. 1-58.

[BGVV14] S. Brazitikos, A. Giannopoulos, P. Valettas, and B.-H. Vritsiou, Geometry of isotropic convex bodies, Mathematical Surveys and Monographs, vol. 196, American Mathematical Society, Providence, RI, 2014.

[DR50] A. Dvoretzky and C. A. Rogers, Absolute and unconditional convergence in normed linear spaces, Proc. Nat. Acad. Sci. U. S. A. 36 (1950), 192-197.

[Gru07] P. M. Gruber, Convex and discrete geometry, Grundlehren der Mathematischen Wissenschaften [Fundamental Principles of Mathematical Sciences], vol. 336, Springer, Berlin, 2007.

[GS05] P. M. Gruber and F. E. Schuster, An arithmetic proof of John's ellipsoid theorem, Arch. Math. (Basel) 85 (2005), no. $1,82-88$.

[Joh48] F. John, Extremum problems with inequalities as subsidiary conditions, Studies and Essays Presented to R. Courant on his 60th Birthday, January 8, 1948, Interscience Publishers, Inc., New York, N. Y., 1948, pp. 187-204.

[LYZ04] E. Lutwak, D. Yang, and G. Zhang, Volume inequalities for subspaces of $L_{p}$, J. Differential Geom. 68 (2004), no. 1, 159-184.

[MP] V. D. Milman and A. Pajor, Isotropic position and inertia ellipsoids and zonoids of the unit ball of a normed n-dimensional space, Geometric Aspects of Functional Analysis: Israel Seminar (GAFA) 1987-88 (J. Lindenstrauss and V. D. Milman, eds.), Vol. 1376, Springer, 1989, pp. 64-104, DOI 10.1007/BFb0090049.

[Nas16] M. Naszódi, Proof of a conjecture of Bárány, Katchalski and Pach, Discrete Comput. Geom. 55 (2016), no. 1, 243-248, DOI 10.1007/s00454-015-9753-3.

[Peł90] A. Pełczyński, Remarks on John's theorem on the ellipsoid of maximal volume inscribed into a convex symmetric body in $\mathbf{R}^{n}$, Note Mat. 10 (1990), no. suppl. 2, 395-410.

[PS91] A. Pełczyński and S. J. Szarek, On parallelepipeds of minimal volume containing a convex symmetric body in $\mathbf{R}^{n}$, Math. Proc. Cambridge Philos. Soc. 109 (1991), no. 1, 125-148.

[Piv10] P. Pivovarov, On determinants and the volume of random polytopes in isotropic convex bodies, Geom. Dedicata 149 (2010), 45-58.

[Sch14] R. Schneider, Convex bodies: the Brunn-Minkowski theory, Second expanded edition, Encyclopedia of Mathematics and its Applications, vol. 151, Cambridge University Press, Cambridge, 2014.

[Sch] R. Schneider, Discrete aspects of stochastic geometry, Handbook of discrete and computational geometry (J. E. Goodman, J. O'Rourke, and C. D. Tóth, eds.), Discrete Mathematics and its Applications (Boca Raton), CRC Press, Boca Raton, FL, 2018. Third edition, pp. 299-329.

[SW08] R. Schneider and W. Weil, Stochastic and integral geometry, Probability and its Applications (New York), SpringerVerlag, Berlin, 2008.

Department of Geometry, Bolyai Institute, University of Szeged, Aradi vértanúk tere 1, 6720 Szeged, Hungary

Email address: fodorf@math.u-szeged.hu

Department of Geometry, Eötvös University, Budapest, Hungary

Email address: marton.naszodi@math.elte.hu

Bolyai Institute, University of Szeged, Aradi vértanúk tere 1, 6720 Szeged, Hungary

Email address: tzarnocz@math.u-szeged.hu 